\title{
Topographical Organization of the Pyramidal Fiber System - Diffusion Tensor MRI of the Human and Rhesus Monkey Brain
}

\author{
Sabine Hofer ${ }^{*}, 1,2$ and Jens Frahm ${ }^{1}$ \\ ${ }^{I}$ Biomedizinische NMR Forschungs GmbH am Max-Planck-Institut für biophysikalische Chemie, 37070 Göttingen, \\ Germany \\ ${ }^{2}$ Bernstein Center for Computational Neuroscience, 37073 Göttingen, Germany
}

\begin{abstract}
The fibers of the pyramidal tract (PT) that connect the precentral and postcentral gyrus with the spinal cord are organized in a topological manner. However, their exact arrangement and orientation in the internal capsule is still a matter of debate. Here, we applied magnetic resonance diffusion tensor imaging and tract tracing techniques to determine and compare the pyramidal fibers from the primary motor and somatosensory system in humans and rhesus monkeys in vivo. The results demonstrate that the pyramidal systems of the human and monkey brain differ in their orientation at the level of the internal capsule, whereas track orientations in the gyrus and brainstem appear similar. In the monkey internal capsule the somatotopic arrangement of PT fibers from the upper and lower extremities form an angle with the left-right axis of $146^{\circ} \pm 23^{\circ}(n=4)$. Thus, fibers from lateral areas of the gyrus are located more anterior-medial to fibers from medial cortical areas. In contrast, in humans the angle is only $50^{\circ} \pm 16^{\circ}(\mathrm{n}=9)$ yielding a more anterior-lateral orientation of PT fibers along the short axis of the internal capsule. These species dependencies may possibly be due to structural constraints of the smaller and differently shaped monkey brain.
\end{abstract}

Keywords: MRI, diffusion tensor imaging, pyramidal fiber, internal capsule, macaque, human.

\section{INTRODUCTION}

In the mammalian brain, the pyramidal tract (PT) is a subset of the massive corticofugal fiber system, which constitutes the main white matter connection between the cerebral cortex and spinal cord. It serves to link higher cortical functions to the voluntary muscular movements of the arms, legs, and torso. Although present at birth, the fiber composition of the PT undergoes several postnatal modifications as the acquisition of motor skills is correlated with a progressive decrease of fiber numbers and coupled with a more widespread distribution of fibers to target areas in the spinal cord $[1,2]$.

Structurally, the PT is composed of more than 1 million fibers arising from the precentral gyrus (primary motor area, about $50 \%$ of fibers) and the postcentral gyrus (primary somatosensory area, about $20 \%$ of fibers). The remaining fibers originate from premotor and parietal areas of the brain. At the level of the medullar pyramid most of the fibers are myelinated with a diameter of 1 to $4 \mu \mathrm{m}$. Single fibers reach a caliber of 5 to $22 \mu \mathrm{m}$ [3]. The fibers from different cortical areas converge into the corona radiata and form a longitudinally oriented fiber bundle of exceptional density in the semioval center [4]. As a consequence, even small lesions of the PT in this compact structure may cause large motor disabilities. The fan-shaped structure of the PT fiber bundle rotates its orientation when proceeding toward the internal

*Address correspondence to this author at the Biomedizinische NMR Forschungs GmbH, 37070 Göttingen, Germany; Tel: +49-551-201-1735; Fax: +49-551-201-1307; E-mail: shofer1@gwdg.de capsule [4] where it travels through the posterior limb following the same somatotopic order as found in the cortex [5]. While the fibers from the lateral cortical parts (upper extremities) are in the more anterior mid-portion of the posterior limb of the internal capsule, the medial projections (lower extremities) occur in its posterior part $[6,1,4]$. Nevertheless, the exact location of PT fibers and the degree of their rotation has been the subject of debates and still remains an open question.

Possible explanations for the uncertainties regarding the PT anatomy result from differences between humans and non-human primates as well as from the use of different experimental approaches ranging from whole brain dissections to lesion studies [4, 6-9] and, more recently, magnetic resonance diffusion tensor imaging (DTI) and virtual fiber tractography [10-14]. For example, tracking of pyramidal motor fibers in stroke patients revealed a somatotopic arrangement of the upper and lower extremities in anteriorposterior direction within the posterior limb of the internal capsule [12]. Conversely, tracking of hand and foot fibers in tumor patients indicated the fibers to be arranged along the left-right axis [11]. In another study, the observation of both topological orientations in a group of subjects was ascribed to interindividual differences in brain anatomy [13]. In fact, although DTI-based fiber tractography allows for a straightforward visualization of PT fibers in healthy humans and monkeys [15-19] as well as in patients with subcortical lesions [20], pertinent studies did not focus on the detailed topological organization of the PT as it passes through the semioval center and internal capsule into the brainstem. 
Technically, DTI exploits directional differences in the mobility or self-diffusivity of water molecules that are caused by differences in the cellular microstructure of the brain tissue [21]. In white matter, diffusion in the direction of a nerve fiber, that is along the axon, is much faster than in the perpendicular direction. Therefore, if axon bundles yield coherently arranged structures that scale to an image voxel, the macroscopically measurable diffusion properties become directionally dependent. When assuming the main diffusion direction to coincide with the direction of the underlying fiber bundle, this information may be exploited for a virtual reconstruction of three-dimensional fiber bundles in the living organism.

The purpose of this work was to further detail the pyramidal fiber system in the human brain and, in view of the existing discrepancies, to compare it to that of the rhesus monkey brain. Moreover, we used the same methodology for both species, that is in vivo DTI and fiber tractography. We were particularly interested in a re-evaluation of the somatotopic arrangement of PT fibers originating from medial to lateral regions of the primary motor and somatosensory cortex, respectively, and their course and relative orientation as they pass through the semioval center and internal capsule into the brainstem.

\section{MATERIAL AND METHODS}

\section{MRI of Human Subjects}

A total of nine right-handed subjects ( 4 female, 5 male; age range 21-47 years) participated in the study. None of them had any history of neurological abnormality. Handedness was assessed in accordance to the hand preference score reported by Oldfield [22]. All subjects gave written informed consent before each MRI examination.

MRI studies were conducted at 2.9 T (Tim Trio, Siemens Healthcare, Erlangen, Germany), using an 8-channel phasedarray head coil. DTI was performed at $2 \mathrm{~mm}$ isotropic resolution using diffusion-weighted single-shot stimulated echo acquisition mode (STEAM) MRI sequences and 5/8 partial Fourier encoding in combination with a projection onto convex subjects (POCS) reconstruction [23,24]. The protocol comprised 24 independent diffusion gradient directions and $b$ values of 0 and $1000 \mathrm{~s} / \mathrm{mm}^{2}$. A total of 50 transverse sections ( $2 \mathrm{~mm}$ thickness) covered the brain. The acquisition time per dataset was approximately 9 minutes. To increase the signal-to-noise ratio, the acquisition was repeated three times. Anatomical images were based on a $T_{1}$-weighted 3D fast low angle shot (FLASH) MRI sequence (repetition time $\mathrm{TR}=11 \mathrm{~ms}$, echo time $\mathrm{TE}=4.9 \mathrm{~ms}$, flip angle $15^{\circ}$ ) yielding an isotropic spatial resolution of $1 \mathrm{~mm}$.

\section{MRI of Rhesus Monkeys}

Four male rhesus macaque (Macaca mulatta, 4 years old) were premedicated using $0.01 \mathrm{mg} \mathrm{kg}^{-1}$ atropin. After induction by intramuscular injection of $15 \mathrm{mg} \mathrm{kg}^{-1}$ ketamine, general anesthesia was maintained by continuous intravenous propofol (10-15 $\left.\mathrm{mg} \mathrm{kg}^{-1}\right)$. For MRI, animals were studied in a supine position with the head centered within the concavity of a 4-channel small shoulder coil using a home-made acrylic head holder $[19,25]$. Animal experiments were approved by national animal welfare authorities, LAVES,
Braunschweig, Germany (reference no. 509.42502/0807.02).

MRI studies of rhesus monkeys employed the same system and sequences as used for humans. However, anatomical images were obtained at $0.5 \mathrm{~mm}$ isotropic resolution and diffusion-weighted STEAM MRI was performed at $1.5 \mathrm{~mm}$ isotropic resolution. Multislice cross-sectional images covering the whole brain (36 contiguous sections at $1.5 \mathrm{~mm}$ thickness) were acquired along a horizontal orientation parallel to the anterior-posterior commissure [as described in 19,25]. The total acquisition time was $28 \mathrm{~min}$.

\section{Fiber Tractography}

First of all, it should be noted that stimulated echoes are insensitive to magnetic field inhomogeneities, so that the geometrically undistorted STEAM images as well as the fiber tracks derived therefrom are spatially congruent to the anatomical images. Without the need for any co-registration or alignment technique which is usually accompanied by a certain degree of blurring, the present work therefore allowed for a direct digital superposition of corresponding data matrices. Before calculation of the diffusion tensor the diffusion-weighted STEAM MRI datasets were interpolated to $1.0 \mathrm{~mm}$ isotropic resolution for human studies and $0.75 \mathrm{~mm}$ for monkey studies, that is half of the original linear voxel dimension or $1 / 8$ th of the voxel size. Estimates of axonal projections were computed by the tensorlines algorithm [26] using 9 seed points per image voxel, that is one central position complemented by two points half way on either side of each of the four spatial diagonals. The DTI analysis relied on software developed in house [27] and was previously applied to map the topography of diverse fiber connections in humans and monkeys [17,19,25].

Tracking terminated when the fractional anisotropy was between 0.08 and 0.15 or the main diffusion directions in consecutive steps differed by more than $15^{\circ}-20^{\circ}$, depending on the individual anatomical organization of PT fibers. In all cases the choice of empirically optimized thresholds and the definition of regions-of-interest (ROIs) were entirely based on existing anatomical knowledge with reference to classical textbooks [2,28].

Reconstruction of the pyramidal fiber system was accomplished with a region-to-region tracking technique using ROIs that were identified on horizontal sections (Fig. 1). A series of sequential start ROIs (colored strips) was placed along the precentral and postcentral gyrus, respectively, and complemented by a target ROI (red bars) inferior to the internal capsule. The seven start ROIs in the respective cortex of either hemisphere covered the full medial-lateral extension of the gyrus in equal parts. Furthermore, to separate the PT from other projections of the large corticofugal fiber system such as the corticopontine and corticobulbar bundles, only tracts were included, which travel the exact course via the posterior limb of the internal capsule into the brainstem.

The procedure resulted in up to 28 PT fiber profiles ( 7 ROIs, two cortices, two hemispheres) traveling from the cortex to the brainstem. In all individuals, anatomical sections at the level of the insular, displaying the caudate nucleus, putamen, and thalamus, were chosen to visualize the more superior part of the internal capsule (best anatomical 


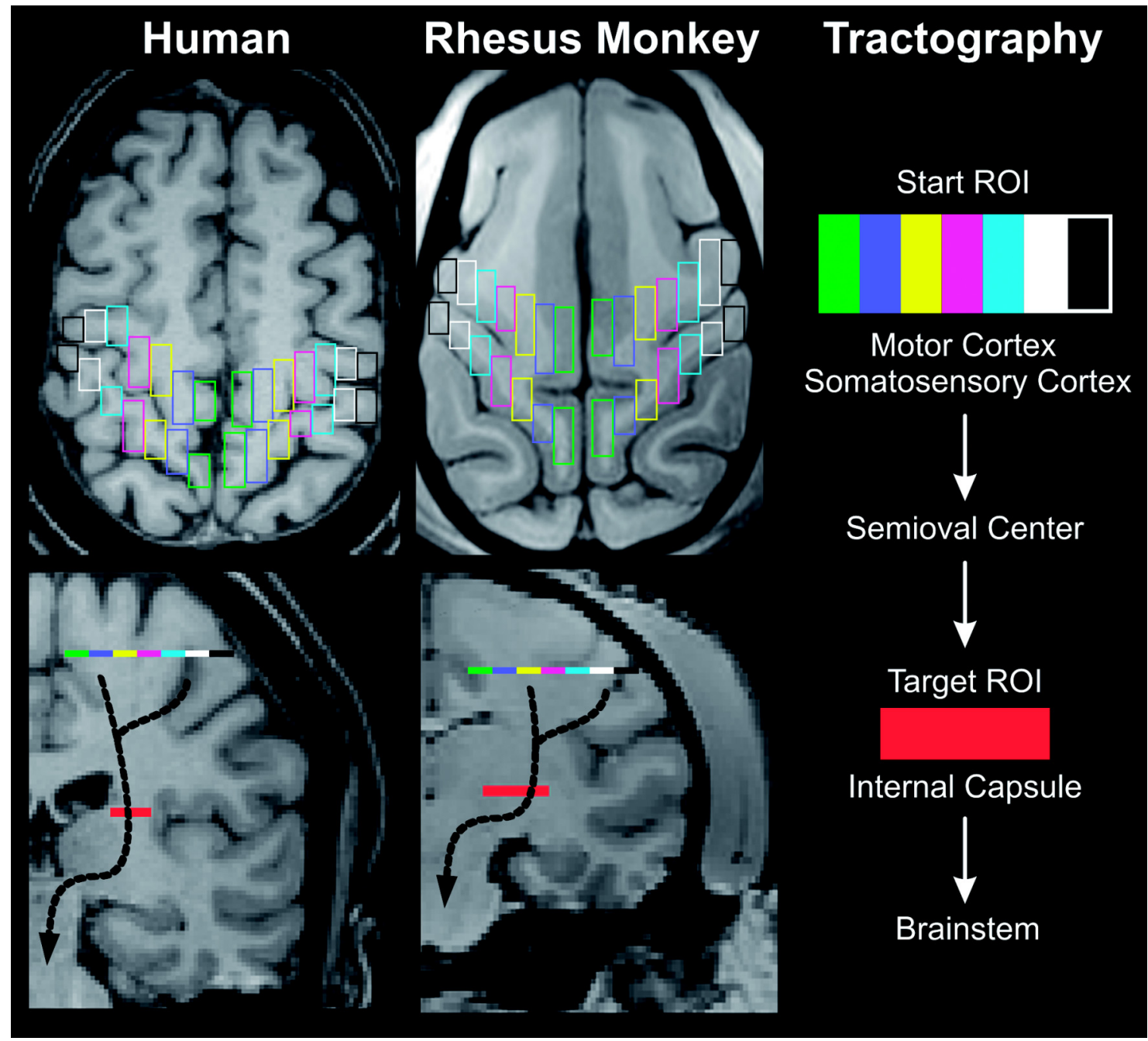

Fig. (1). Region-to-region tracking of pyramidal fibers from the motor and somatosensory cortex in the human and rhesus monkey brain. Cortical start regions are color-coded for medial (green) to lateral (black) parts of the gyri on (top) horizontal and (bottom) coronal images. The common target ROI covering the ipsilateral internal capsule is coded in red.

view of the posterior limb) and to characterize the topological orientation of the respective fiber profiles. Because of known methodological limitations of DTI-based fiber tractography, it was not always possible to track all fibers in every subject. In particular, connections to the most lateral parts of the motor and somatosensory cortex were more difficult to reconstruct, as respective fibers cross other pronounced white matter tracks such as the superior longitudinal fasciculus.

Centers of gravity were obtained for all partitioned motor and somatosensory fiber profiles (Image J) and used to determine their topological order and angular rotation in the internal capsule. The latter values were tested for significance (student t-test) at a threshold of $p<0.05$ (Microsoft Excel). We defined the left-right axis as $0^{\circ}$ and the anteriorposterior axis along the interhemispheric fissure as $90^{\circ}$.

\section{RESULTS}

In this study, we were able to reproducibly track the pyramidal system of all subjects in both the left and right hemisphere and in a manner consistent with anatomical data. Typical findings are shown for a single human subject and rhesus monkey (Figs. 2-6). Reconstructed fibers in human and monkey brain that originate from neighboring areas of the precentral motor cortex and postcentral sensory cortex path via the semioval center through the posterior limb of the internal capsule into the brainstem. Because DTI is blind to the direction of the fiber bundles (afferent or efferent), the tracks include both ascending and descending fibers. On the other hand, while many descending fibers terminate in or send collateral branches to a number of supraspinal structures such as the thalamus, red nucleus, and pontine nucleus, tractography of these fibers was excluded.

In both the human and monkey brain, PT fibers exhibit a fan-like arrangement, which opens toward the cortex, closes when approaching the internal capsule, and opens again in the brainstem (Fig. 2). In this respect, fibers from the primary somatosensory cortex behave similar to those originating from the primary motor cortex in both species (Fig. 3). In the semioval center, internal capsule, and brainstem, sensory PT fibers always follow the motor PT fibers in a parallel but more posterior position. Moreover, the topographical arrangement of fibers from the precentral and postcentral gyrus (compare Fig. 1) is preserved along the entire path of the PT 


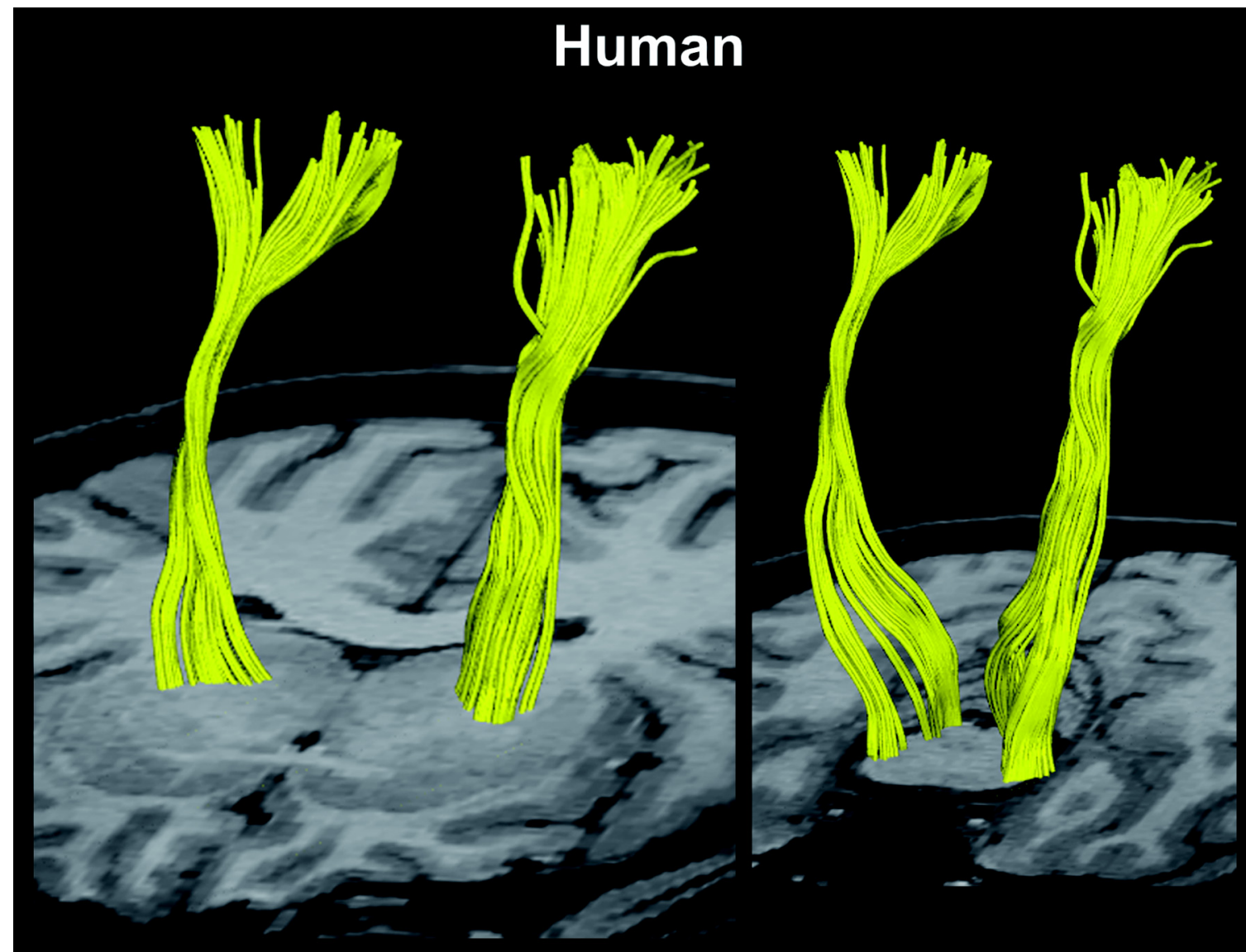

\section{Rhesus Monkey}

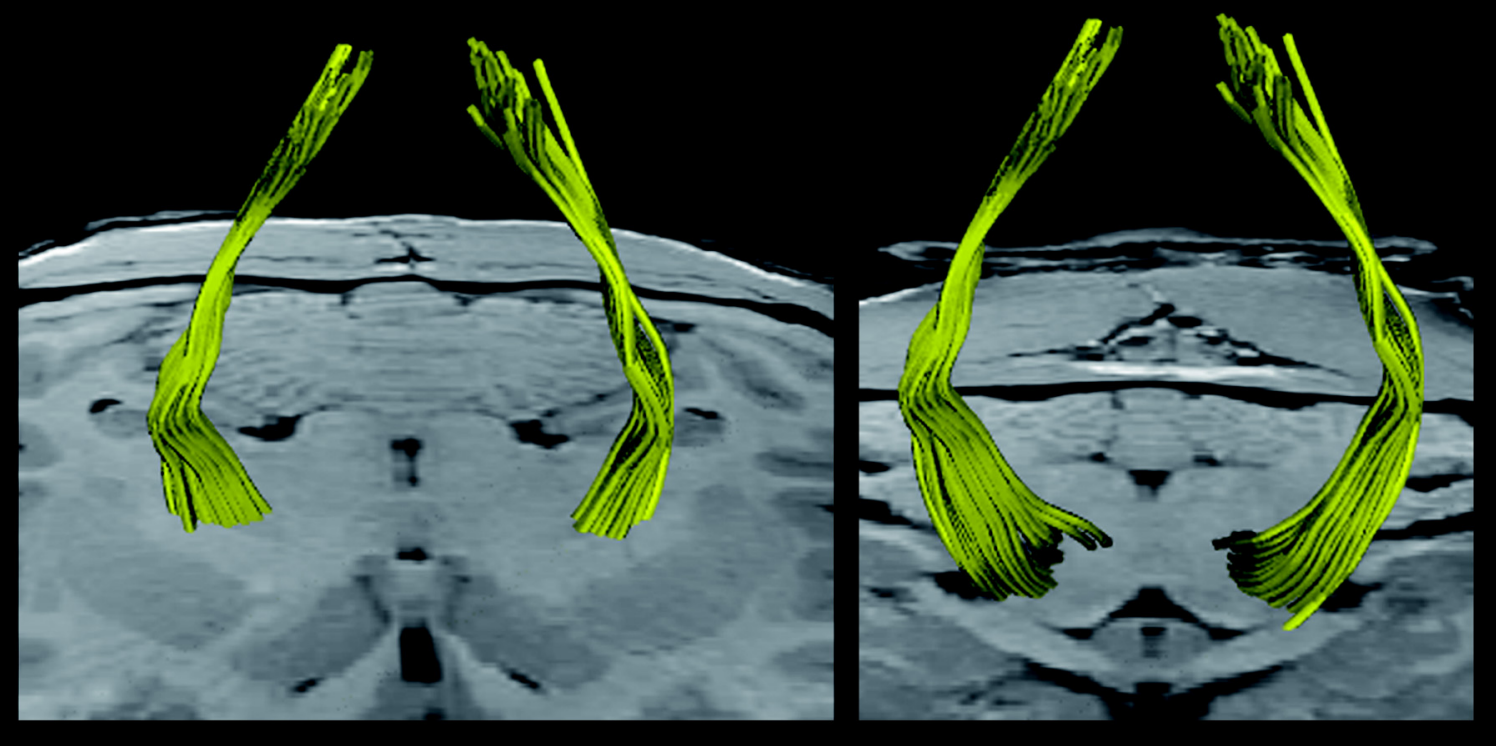

Fig. (2). A subset of pyramidal motor fibers originating from the yellow start ROIs (Fig. 1) in the left- and right-hemispheric motor cortices of the human and rhesus monkey brain at the level of the (left) internal capsule and (right) brainstem.

in both species, that is in the semioval center (Fig. 4), internal capsule (Fig. 5), and brainstem (Fig. 6). The color-coded fiber profiles do not represent functionally defined cortical areas, but were deliberately chosen as a geometric partitioning of the gyrus that should allow for both a clear assessment of fiber composition and topology in different subjects and a meaningful comparison between humans and monkeys. In fact, in both species fiber bundles from medial parts of the cortex (green and blue, near the interhemispheric fissure; Figs. 4-6) are found in more posterior locations of the internal capsule than fibers from lateral parts of the cortex (light blue, white, black; Figs. 4-6). With respect to the twist of the $\mathrm{PT}$, fibers from more lateral parts of the gyrus rotate into more anterior positions on their route to the internal capsule. 


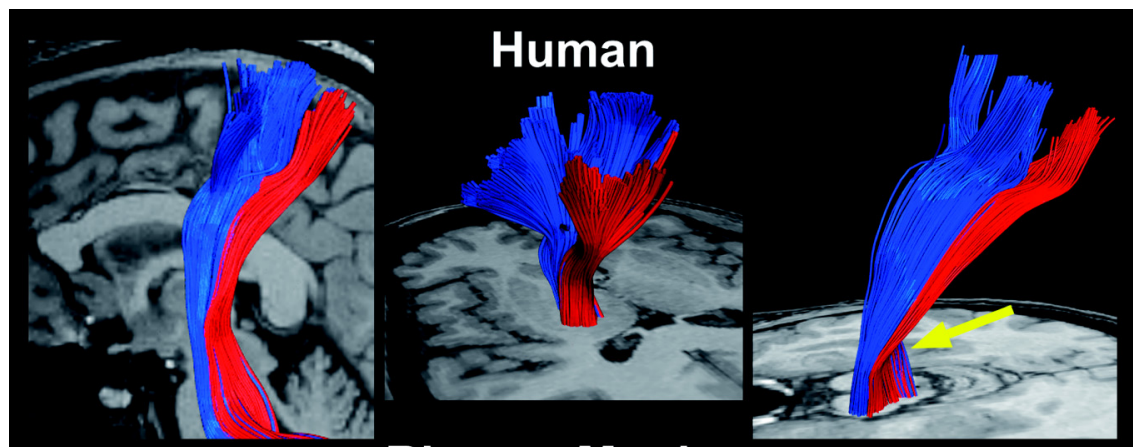

Rhesus Monkey

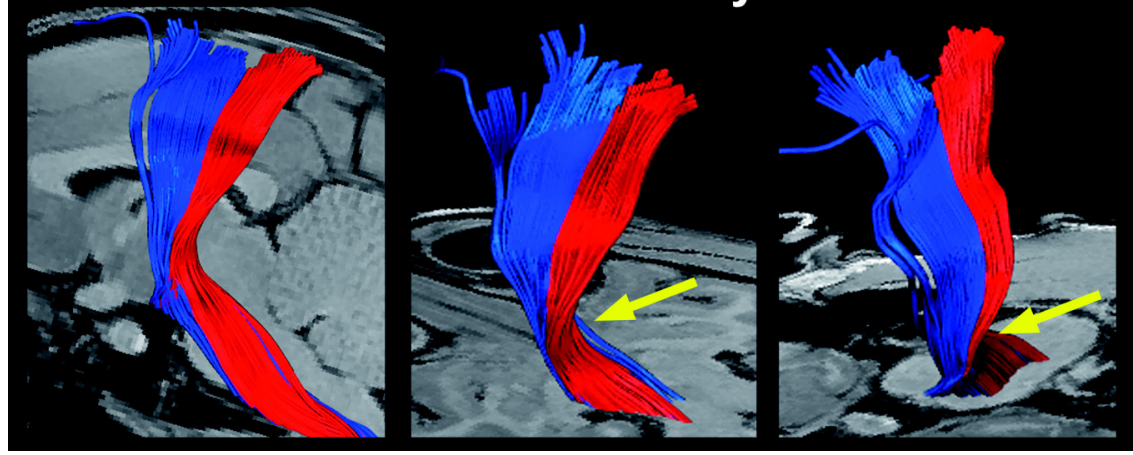

Fig. (3). Pyramidal motor (blue) and somatosensory fibers (red) in the human and rhesus monkey brain in (left) sagittal and (middle, right) oblique horizontal orientations. The fibers rotate along the left-right axis (arrow) as shown at the level of the (middle) internal capsule and (right) brainstem.

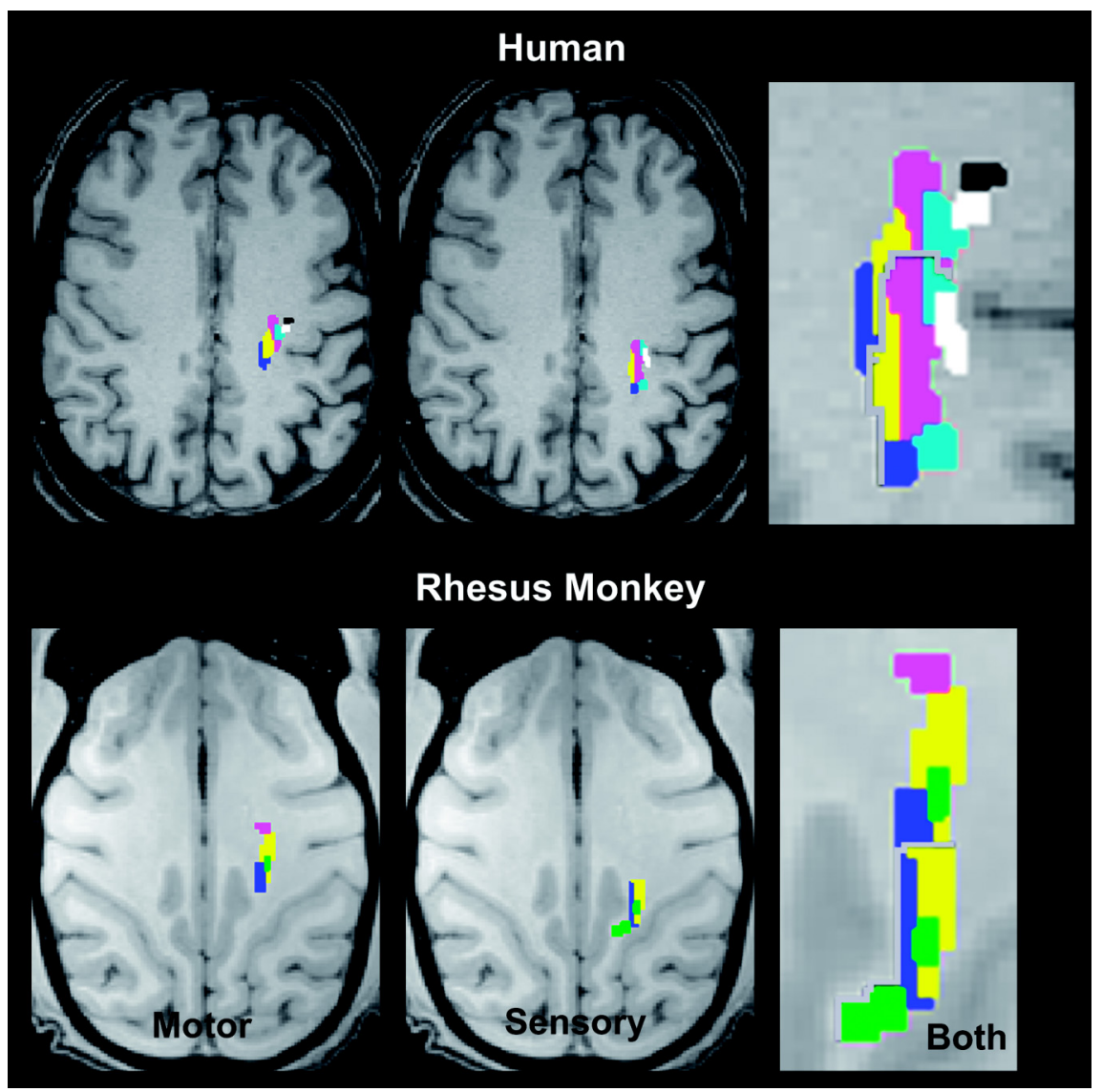

Fig. (4). Pyramidal motor and somatosensory fiber profiles of the human and rhesus monkey brain in the semioval center (colors refer to ROIs in Fig. 1). The enlarged views represent overlays of motor and somatosensory fibers with the gray line indicating the border between both systems. 


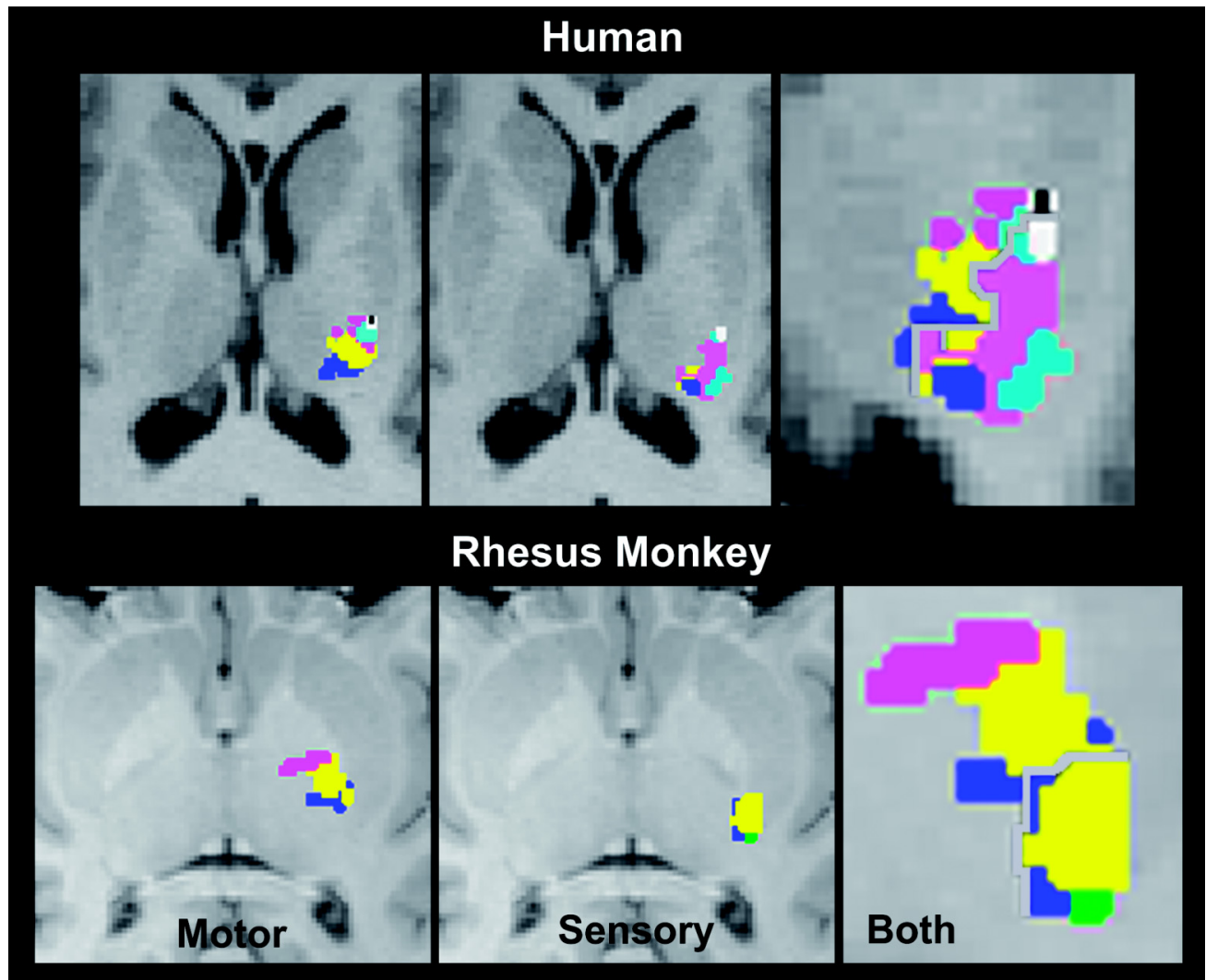

Fig. (5). Pyramidal motor and somatosensory fiber profiles of the human and rhesus monkey brain in the internal capsule (posterior part of the posterior limb, colors refer to ROIs in Fig. 1). The enlarged views represent overlays of motor and somatosensory fibers with the gray line indicating the border between both systems.
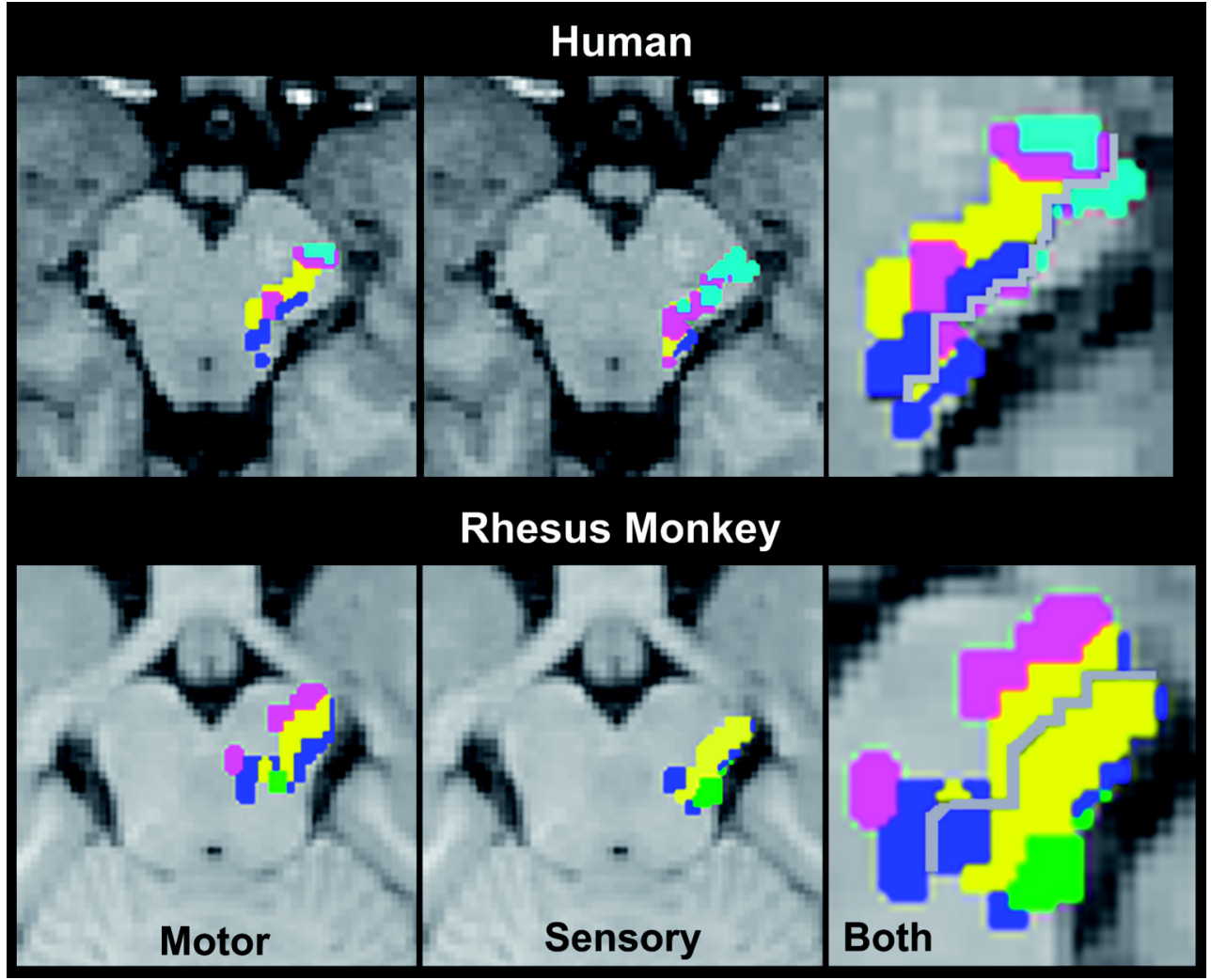

Fig. (6). Pyramidal motor and somatosensory fiber profiles of the human and rhesus monkey brain in the brainstem (colors refer to ROIs in Fig. 1). The enlarged views represent overlays of motor and somatosensory fibers with the gray line indicating the border between both systems. 
A comparison of the PT in the human and monkey internal capsule revealed significant differences (Fig. 5). Apart from the fact that in the monkey brain the PT rotation already begins in more ventral parts, the angle formed by the entirety of motor and somatosensory PT fibers with the leftright axis is $50^{\circ} \pm 16^{\circ}$ in humans, but $146^{\circ} \pm 23^{\circ}$ in monkeys $(\mathrm{P}<0.05$; mean $\pm \mathrm{SD})$. This finding can also qualitatively be observed in three-dimensional views of the fiber tracks (Fig. 3). In more detail, in human (monkey) brain the rotation amounts to $47.9^{\circ} \pm 13^{\circ}\left(138^{\circ} \pm 26^{\circ}\right)$ for the motor PT and $52.1^{\circ} \pm 19^{\circ}\left(152^{\circ} \pm 21^{\circ}\right)$ for the somatosensory PT.

In contrast to the compact form of the fiber tracks in the semioval center and internal capsule, the bundles seem to expand in the brainstem. In both humans and monkeys, the motor and somatosensory PT fibers stretch along the horizontal axis of the brainstem and travel into deeper brain parts. Motor fibers are kept in more anterior parts of the brainstem and sensory fibers posteriorly. In addition, the PT still maintains its somatotopic ordering with fibers from medial cortical areas (green and blue, Fig. 6) in more posterior and lateral fibers (light blue, Fig. 6) in more anterior locations of the brainstem.

A schematic outline and geometric representation of the topographically organized PT from the cortex to the brainstem (Fig. 7) highlights the general similarity of the pyramidal system in the human and rhesus monkey brain as well as the pronounced quantitative differences in the internal capsule.

\section{DISCUSSION}

Using DTI-based fiber tractography, we compared the pyramidal motor and somatosensory fiber system in humans and rhesus monkeys in vivo. In particular, we assessed the location, topology, and rotation of fibers representing neighboring cortical projection areas in the semioval center, internal capsule, and brainstem.
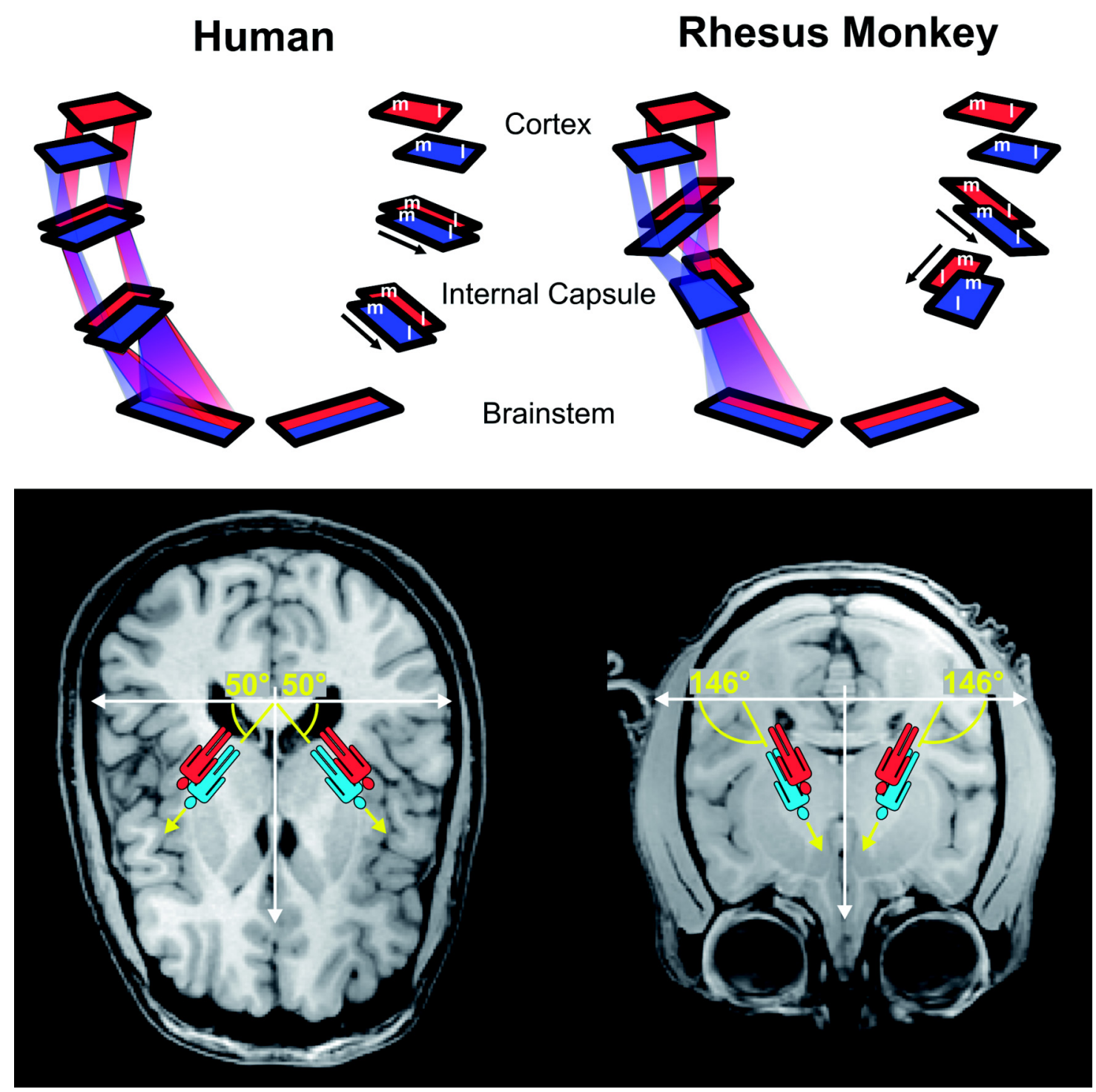

Fig. (7). Frontal views of (top) a schematic outline and (bottom) geometric representation of the pyramidal fiber system in the human and rhesus monkey brain (blue $=$ motor fibers, red $=$ somatosensory fibers). The medial $(\mathrm{m})$ and lateral (l) fiber bundles exhibit a somatotopic arrangement which in the posterior limb of the internal capsule is rotated along the left-right axis by $50^{\circ}$ in humans and $146^{\circ}$ in monkeys. 
First of all, the results confirm previous findings that place the PT of the primary motor and primary sensory system into the posterior limb of the internal capsule, in humans as well as in monkeys $[6,28,29]$. Secondly, we observed a rotation of the PT on its way from the cortex through the semioval center and internal capsule into the brainstem. Thirdly, the principle arrangement of PT fibers turns out to be rather similar in both species. This applies to the fact that the somatosensory PT tightly parallels the motor PT and also holds true for the preservation of a somatotopic order down to the brainstem. Fourthly, however, we found pronounced species-dependent differences in the orientation of the primary motor and somatosensory PT in the internal capsule. In the monkey brain the PT rotation is almost three times larger than in the human brain and begins shortly after the fibers leave their cortical projection area.

It seems as if the descending fibers of the PT must rotate in order to maintain the somatotopic order while continuing through the brainstem into the spinal cord. One may speculate that this arrangement is a plausible consequence of a structural optimization that ensures a rapid and efficient arrival in the target region - with fibers conducting information for the upper parts of the body ending in more anterior portions of the brainstem (torso followed by the arms, hands, and feet). To achieve this topological orientation, the PT in monkeys begins to rotate earlier, that is ventrally in the upper part of the semioval center. Because the monkey brain is smaller than the human brain and more flat in the ventraldorsal extension, one may further speculate that these structural constraints are responsible for a more pronounced PT rotation than in humans.

In this work, we used a DTI technique not hampered by geometric distortions due to tissue susceptibility differences $[23,24]$. Although this offers direct spatial congruence of the reconstructed PT fiber arrangement with anatomical MRI data sets, the tractography technique still suffers the same general problems from crossing or "kissing" (parallel) fibers as discussed by many authors before. Thus, it was not always possible to obtain fiber profiles for all ROIs of the sensorimotor gyri. This especially applies to fibers originating from lateral parts that have to cross the superior longitudinal fasciculus. A further limitation may arise from the lack of a functional definition of the start ROIs. However, the choice of a geometric definition with equal and directly neighboring ROIs covering the extended gyri on an optimized horizontal section guaranteed for an unbiased comparability between both subjects and species. It certainly served to answer our key questions about the topological PT fiber representation and its change of orientation within the internal capsule.

In conclusion, we found qualitative similarity of the pyramidal fiber system in the human and monkey brain in vivo, but also marked differences in the detailed organization of PT fibers in the posterior limb of the internal capsule. The rotation of the somatotopic arrangement observed for fibers from both the primary motor and somatosensory cortex is three times larger in monkeys than in humans and may reflect structural constraints of the monkey head.

\section{REFERENCES}

[2] Tamraz JC, Comair Y. Atlas of regional anatomy of the brain using MRI with functional correlations. New York: Springer-Verlag 2006.

[3] Yagishita A, Nakano I, Oda M, Hirano A. Location of the corticospinal tract in the internal capsule at MR imaging. Radiology 1994; 191: 455-60.

[4] Ebeling U, Reulen HJ. Subcortical topography and proportions of the pyramidal tract. Acta Neurochir (Wien) 1992; 118: 164-71.

[5] Penfield W, Rasmussen T. The cerebral cortrex of man: a clinical study of localization of function. New York: Macmillan 1950.

[6] Ross ED. Localization of the pyramidal tract in the internal capsule by whole brain dissection. Neurology 1980; 30: 59-64.

[7] Fromm C, Evarts EV. Pyramidal tract neurons in somatosensory cortex: central and peripheral inputs during voluntary movement. Brain Res 1982; 22: 186-191.

[8] Fromm C, Wise SP, Evarts EV. Sensory response properties of pyramidal tract neurons in the precentral motor cortex and postcentral gyrus of the rhesus monkey. Exp Brain Res 1984; 54: 177-185.

[9] Morecraft RJ, Herrick JL, Stilwell-Morecraft KS, et al. Localization of arm representation in the corona radiata and internal capsule in the non-human primate. Brain 2002; 125: 176-198.

[10] Parker GJM, Stephan KE, Barker GJ, et al. Initial demonstration of in vivo tracing of axonal projections in the macaque brain and comparison with the human brain using diffusion tensor imaging and fast marching tractography. Neuroimage 2002; 15: 797-809.

[11] Holodny AI, Gor DM, Watts R, Gutin PH, Ulug AM. Diffusiontensor MR tractography of somatotopic organization of corticospinal tracts in the internal capsule: initial anatomic results in contradistinction to prior reports. Radiology 2005; 234: 649-53.

[12] Lee JS, Han MK, Kim SH, Kwon OK, Kim JH. Fiber tracking by diffusion tensor imaging in corticospinal tract stroke: topographical correlation with clinical symptoms. Neuroimage 2005; 26: 771-6.

[13] Ino T, Nakai R, Azuma T, Yamamoto T, Tsutsumi S, Fukuyama H. Somatotopy of corticospinal tract in the internal capsule shown by functional MRI and diffusion tensor images. Neuroreport 2007; 18: 665-68.

[14] Dauguet J, Peled S, Berezovskii V, et al. Comparison of fiber tracts derived from in-vivo DTI tractography with 3D histological neural tract tracer reconstruction on a macaque brain. Neuroimage 2007; 15: 530-38.

[15] Mori S, Kaufmann WE, Davatzikos C, et al. Imaging cortical association tracts in the human brain using diffusion-tensor-based axonal tracking. Magn Reson Med 2002; 2: 215-23.

[16] Wakana S, Jiang H, Nagae-Poetscher LM, van Zijl PC, Mori S, Fiber tract-based atlas of human white matter anatomy. Radiology 2004; 230: 77-87.

[17] Hofer S, Frahm J. Topography of the human corpus callosum revisited - comprehensive fiber tractography using diffusion tensor magnetic resonance imaging. Neuroimage 2006; 32: 989-94.

[18] Schmahmann JD, Pandya DN, Wang R, et al. Association fibre pathways of the brain: parallel observations from diffusion spectrum imaging and autoradiography. Brain 2007; 130: 630-53.

[19] Hofer S, Frahm J. In vivo mapping of fiber pathways in the rhesus monkey brain. Open Med Imaging J 2008; 2: 32-41.

[20] Kunimatsu A, Aoki S, Masutani Y, Abe O, Mori H, Ohtomo K Three-dimensional white matter tractography by diffusion tensor imaging in ischaemic stroke involving the corticospinal tract. Neuroradiology 2003; 45: 532-535.

[21] Basser PJ, Pierpaoli C. Microstructural and physiological features of tissues elucidated by quantitative diffusion-tensor MRI. J Magn Reson B 1996; 111: 209-19.

[22] Oldfield RC. The assessment and analysis of handedness: the Edinburgh inventory. Neuropsychologia 1971; 9: 97-113.

[23] Nolte UG, Finsterbusch J, Frahm J. Rapid isotropic diffusion mapping without susceptibility artifacts: whole brain studies using diffusion-weighted single-shot STEAM MR imaging. Magn Reson Med 2000; 44: 731-36.

[24] Rieseberg S, Merboldt KD, Küntzel M, Frahm J. Diffusion tensor imaging using partial Fourier STEAM MRI with projection onto convex subsets reconstruction. Magn Reson Med 2005; 54: 486-90.

[25] Hofer S, Merboldt K-D, Tammer R, Frahm J. Rhesus monkey and human share a similar topography of the corpus callosum as revealed by diffusion tensor MRI in vivo. Cereb Cortex 2008; 18: 1079-84. 
[26] Weinstein D, Kindlmann G, Lundberg E. Tensorlines: advectiondiffusion based propagation through diffusion tensor fields. In Proc IEEE Vis 1999; 249-53.

[27] Küntzel M. Parallele Datenakquisition zur Beschleunigung diffusions- gewichteter Kernspintomographie mit stimulierten Echos [Parallel data acquisition for diffusion-weighted MRI using stimulated echoes]. Dissertation, Georg-August-Universität, Göttingen.
Online access of the University library. Available from: http: //webdoc.sub.gwdg.de/diss/2007/kuentzel/kuentzel.pdf

[28] Schmahmann JD, Pandya DN. Fiber Pathways of the Brain. Oxford University Press 2006

[29] Zarei M, Johansen-Berg H, Jenkinson M, Ciccarelli O, Thompson AJ, Matthews PM. Two-dimensional population map of cortical connections in the human internal capsule. J Magn Reson Imaging 2007; $25: 48-54$.

(C) Hofer and Frahm; Licensee Bentham Open.

This is an open access article licensed under the terms of the Creative Commons Attribution Non-Commercial License (http://creativecommons.org/licenses/bync/3.0/) which permits unrestricted, non-commercial use, distribution and reproduction in any medium, provided the work is properly cited. 